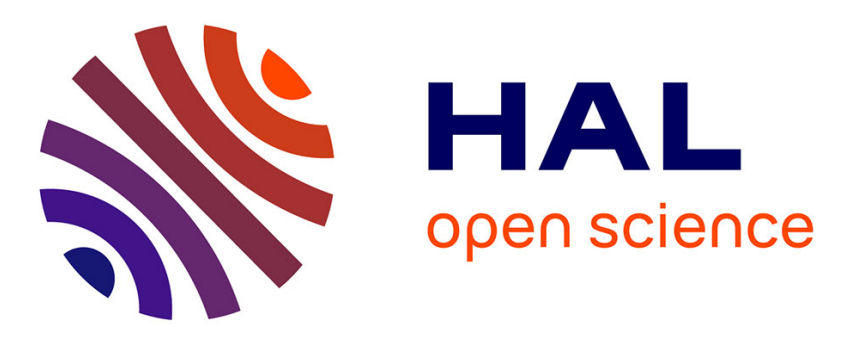

\title{
The enpp4 ectonucleotidase regulates kidney patterning signalling networks in Xenopus embryos
}

Karine Massé, Surinder Bhamra, Christian Paroissin, Lilly Maneta-Peyret, Eric Boué-Grabot, Elizabeth Jones

\section{- To cite this version:}

Karine Massé, Surinder Bhamra, Christian Paroissin, Lilly Maneta-Peyret, Eric Boué-Grabot, et al.. The enpp4 ectonucleotidase regulates kidney patterning signalling networks in Xenopus embryos. 2021. hal-03221080

\author{
HAL Id: hal-03221080 \\ https://hal.science/hal-03221080
}

Preprint submitted on 7 May 2021

HAL is a multi-disciplinary open access archive for the deposit and dissemination of scientific research documents, whether they are published or not. The documents may come from teaching and research institutions in France or abroad, or from public or private research centers.
L'archive ouverte pluridisciplinaire HAL, est destinée au dépôt et à la diffusion de documents scientifiques de niveau recherche, publiés ou non, émanant des établissements d'enseignement et de recherche français ou étrangers, des laboratoires publics ou privés. 


\section{The enpp4 ectonucleotidase regulates kidney patterning}

\section{signalling networks in Xenopus embryos}

5

* Corresponding author: Karine Massé, karine.masse@u-bordeaux.fr

${ }^{1}$ School of Life Sciences, Warwick University, Coventry, CV47AL, U.K.

France

${ }^{3}$ CNRS, Institut des Maladies Neurodégénératives, UMR 5293, F-33000 Bordeaux, France. UMR CNRS 5142, 64013 Pau cedex, France

${ }^{5}$ Université de Bordeaux, CNRS, Laboratoire de Biogenèse Membranaire UMR 5200, F-33800

Villenave d'Ornon, France

2Université de Bordeaux, Institut des Maladies Neurodégénératives, UMR 5293, F-33000 Bordeaux,

${ }^{4}$ Université de Pau et des Pays de l'Adour, Laboratoire de Mathématiques et de leurs Applications - 


\section{Abstract}

29 The enpp ectonucleotidases regulate lipidic and purinergic signalling pathways by controlling the 30 extracellular concentrations of purines and bioactive lipids. Although both pathways are key regulators 31 of kidney physiology and linked to human renal pathologies, their roles during nephrogenesis remain 32 poorly understood. We previously showed that the pronephros was a major site of enpp expression and 33 now demonstrate an unsuspected role for the conserved vertebrate enpp4 protein during kidney 34 formation in Xenopus. Enpp4 over-expression results in ectopic renal tissues and, on rare occasion, 35 complete mini-duplication of the entire kidney. Enpp4 is required and sufficient for expression of the pronephric markers and regulates the expression of RA, Notch and Wnt pathway members. Enpp4 is a membrane protein that binds, without hydrolyzing, phosphatidylserine and its effects are mediated by the receptor s1pr5, although not via the generation of S1P. Finally, we propose a novel and surprising non-catalytic mechanism by which lipidic signalling regulates nephrogenesis.

Keywords: Enpp4, ectonucleotidase, s1p5r, lipidic signalling, kidney development, pronephros, patterning, organogenesis, Xenopus. 
Introduction

52

Vertebrate kidney organogenesis is orchestrated by numerous signaling pathways and transcription factors regulating the proliferation and differentiation of diverse cell types to form the functional kidney. Despite the differences in complexity and organization of the three vertebrate kidneys, pronephros, mesonephros and metanephros, there is a remarkable conservation of molecular mechanisms during their development ${ }^{1}$. The pronephros, the functional embryonic kidney in amphibians, is a simple, easily accessible organ, which displays structural similarities to the other more complex kidney forms. Therefore, it has become an ideal model system to study molecular regulation during nephrogenesis and renal pathologies $2,3,4,5$.

In mammals, lipidic and purinergic pathways regulate metanephric physiology and their deregulation has been linked to renal pathologies $6,7,8,9$. However, their potential roles during renal development have not been fully established, although the bioactive lipid sphingosine-1-phosphate (S1P) has been implicated during kidney branching ${ }^{10}$. Purines, mostly ATP and its derivatives, and S1P and lysophosphatidic acid (LPA) can function as extracellular ligands for G protein-coupled cell surface receptors ${ }^{11,12}$. Their availability for these receptors, in the extracellular space, is regulated by the activities of several membrane-bound enzymes, such as the ectonucleotidases, which are major regulators of renal health and disease 13, 14. The enpp (ectophosphodiesterase/nucleotide phosphohydrolase) proteins, which belong to the ectonucleotidase subfamily, are key regulators of both purinergic and lipidic signalling pathways with their dual enzymatic activities of hydrolyzing purines and generating S1P and LPA bioactive lipids ${ }^{15}$. We have demonstrated that the pronephros is the major site of expression for the amphibian enpp genes family, in particular enpp4 is highly expressed in Xenopus laevis pronephric tubules ${ }^{16}$. These data provided the first temporal and spatial embryonic expression profile for this evolutionally conserved enzyme which remains functionally poorly understood ${ }^{17,18,19}$. In the present study, we investigated the function of enpp4 during pronephric development.

We demonstrate that enpp4 function is crucial during kidney formation. While its knock-down leads to kidney formation defects, the over-expression of wild type enpp4, but not an inactive enzymatic protein, induces the formation of ectopic pronephroi characterized mostly by the presence of proximal tubule markers but in rare occasion of more distal tubule markers. These effects are mediated by the lipidic receptor s1pr5 and we also show that enpp4 specifically binds to phosphatidylserine, implying a role for bioactive lipids in pronephrogenesis. Finally, we provide evidence that enpp4 misexpression alters the expression of members of the Notch, Wnt and RA signalling pathways and we propose a model for the mechanisms of action for enpp4 and lipidic signalling in kidney development. 
Results

\section{Over-expression of enpp4 results in ectopic pronephric tubules formation}

To analyze potential functional roles of enpp4 during pronephros development, we first undertook a gain of function approach by performing immunostaining with pronephric tubules specific antibodies ${ }^{20}$ on stage 41 embryos (Fig. 1A-O, and Supplementary Table 1 for raw data and statistical analyses). Enpp4 over-expression altered proximal pronephric tubules formation, in nearly $50 \%$ of the analysed embryos and induced ectopic (23\%) and enlarged (18\%) regions of the $3 \mathrm{G} 8$ staining domain ( $\mathrm{n}=91$, Fig. 1B-E

; Supplementary Table 1). Distal tubules were less affected, with $31 \%$ of the analysed embryos displaying abnormal $4 \mathrm{~A} 6$ staining. Ectopic $4 \mathrm{~A} 6$ staining was rare $(2 \%)$, with enlarged more distal tubule staining being the predominant phenotype (20\%). Enpp4-induced phenotypes are significantly different compared to those of LacZ controls (3G8: $p<0.001$ and 4A6: $p<0.05)$. Ectopic pronephroi were observed only when injections were performed into regions fated to become the lateral region of embryos (V2 blastomere) (Supplementary Fig. 1A). Enpp4 over-expressing embryos displaying ectopic $3 \mathrm{G} 8$ staining $(n=5)$ were analyzed by transverse section. 8 of the 9 ectopic tubules sectioned had epithelial tubule structure complete with a lumen (Fig. 1F-J), which were similar to normal pronephric tubule structure (Fig. 1K-L). Similar significant renal phenotypes were observed following mouse Enpp4 mRNA injection $(p<0.001, n=63$; Fig. 1M). However, no ectopic pronephric tubules were observed upon overexpression of enpp4 mutant constructs containing a point mutation in the putative catalytic domain (T72A, T72S) or metal cation binding domain (D36N, D189N) (Fig. 1N,O) ${ }^{16}$. These data suggest that ectopic proximal pronephric tissues formation caused by enpp4 over-expression depends on its catalytic activity.

108

\section{Over-expression of enpp4 disturbs proximal-distal patterning of pronephros}

110 To further investigate this phenotype, embryos injected with enpp4 mRNA were examined at stage 37

111 by whole mount in situ hybridization using pronephric specific markers, s/c5a1.1, s/c12a1, clcnkb and 112 gata3, which mark the various proximal/distal tubule segments ${ }^{21,22}$ (Fig. $1 \mathrm{~A}$ and P-S, see also 113 Supplementary Table 1). Interestingly, enpp4 overexpressing embryos showed ectopic and enlarged 114 staining of slc5a1.1 (proximal tubule marker, Ectopic 30\%, Enlarged 14\%; $n=57 ; p<0.001$ ) and s/c12a1 115 (marker of intermediate tubules, Ectopic 17\%, Enlarged 25\%; $n=57, p<0.001$ ) domains (Fig. 1P-Q).

116 Injection of enpp4 mRNA failed to induce any separate ectopic clcnkb expression although the normal 117 domain of expression (intermediate and distal tubule) was somewhat enlarged on the injected side $118(19 \%, \mathrm{n}=58, \mathrm{p}<0.01$; Fig. 1R). The gata3 expression domain (distal and collecting tubules) was 119 relatively normal, although its anterior limit of expression, determined relative to the somite number, was 
120 slightly more posterior in more than half of the injected embryos ( $58 \%, n=43, p<0.001$; Fig. 1S). This

121 might reflect a change in anterior/posterior patterning induced by enpp4 over-expression.

122 Injection of enpp4 mRNA induced enlarged and reduced expression domains of both glomus marker

$123 w t 1$ and nphs1 at stage 33/34 but ectopic glomus staining was only observed in rare cases (Fig. 1T-U;

124 Supplementary Table 1A). Although the statistical significance of these phenotypes was demonstrated

125 (Supplementary Table 1B), we were not able to conclude on the exact enpp4 effects on this structure.

126 Taken together, the results demonstrate that enpp4 mRNA injection altered pronephros formation,

127 leading to enlarged expression domains of markers of the entire tubule segments and to ectopic

128 pronephric structures containing mostly domains of proximal and, in rare occasions, distal tubules

129 marker genes.

130

131 Over-expression of enpp4 up-regulates early kidney markers expression without altering 132 mesoderm formation

133 Embryos injected with enpp4 mRNA were also examined by whole mount in situ hybridization using 134 early pronephros anlagen markers $i r x 1, I h \times 1$, pax $8^{23,24}$ and compared to LacZ mRNA injected embryos 135 (See Supplementary Table 1). At stage 28, expression of both $\operatorname{lh} \times 1(61 \%, \mathrm{n}=51)$ and $\operatorname{pax} 8(70 \%, \mathrm{n}=$ $13650)$ was significantly $(p<0.001)$ expanded especially in posterior parts of pronephric anlagen, with areas 137 of intense staining consistent with that of the more anterior presumptive tubules (Fig. 1V-W). At early 138 neurula stages (Fig.1X-Y), the expansion of pax8 $(17 \%, \mathrm{n}=70)$ expression domain was also observed 139 (Fig.1Y). Furthermore, ectopic pax8 expression was also induced following enpp4 RNA injection at both 140 stages analysed (neurula stage, 21\%; stage 28, 2\%; $p<0.001$; Supplementary Table 1B). Lhx1 141 expression was also altered at early neural stages, but no ectopic Lhx1 expression was observed (Fig. 142 1X, Supplementary Table 1B). Furthermore, irx1 expression domain was not altered following enpp4 143 overexpression (Supplementary Fig. 1B).

144 Since normal somite development is a prerequisite for pronephros development, enpp4 RNA injected 145 embryos were analyzed by whole mount in situ hybridization using the muscle marker myh4 at stage 146 33/34. The expression pattern was normal in all injected embryos $(n=55$; Supplementary Fig. 1C;

147 Supplementary Table 1). Enpp4 overexpression did not also alter the expression of pan-mesoderm 148 marker, xbra at stage $10.5(n=46$; Supplementary Fig. 1D and Supplementary Table 1). Therefore, we 149 conclude that, enpp4 mRNA injection had no gross effects on mesoderm induction per se or on somite 150 development.

\section{Morpholino knockdown of enpp4 results in smaller pronephros formation}

153 To determine whether enpp4 is required for normal pronephros development, we have undertaken a 154 loss of function approach using two specific anti-sense morpholino oligonucleotides (MOs) (Fig. 2, 
155 Supplementary Fig. 2A-C for specificity and efficiency of the MOs and Supplementary Table 2). The 156 overall morphology of the embryos appeared normal in enpp4 morphants. Moreover, the expression 157 pattern of the somitic myh4 and early mesoderm xbra markers was unaffected in enpp4 MO1 injected 158 embryos (myh4 100\%, $\mathrm{n}=47$ and xbra 84\%, $\mathrm{n}=19$; Supplementary Fig. 2D-E) suggesting that any 159 kidney phenotypes observed in enpp4 morphants are not due to general mesoderm defects.

160 Injection of enpp4 MO1 resulted in a significant reduction of expression of both $3 G 8(65 \%, p<0.001)$ and 1614 A6 $(28 \%, p<0.05)(n=107$, Fig. 2A-B, Supplementary Table 2), indicating that a smaller pronephros 162 had formed. A similar phenotype was observed following enpp4 MO2 injection (3G8 49\%, 4A6 24\%, $\mathrm{n}=$ 163 87; Supplementary Fig. 2F-G) and was worsened when both MO were injected together (Fig. 2C). These 164 results indicate that enpp4 is required for both proximal and distal pronephric tubule development.

165 Ennp4 knock-down in embryos showed a significant reduced expression of s/c5a1.1 (58\%, $\mathrm{n}=64$, $166 \mathrm{p}<0.001)$ and s/c12a1 (56\%, $\mathrm{n}=75, \mathrm{p}<0.01)$ with MO1 (Fig. 2D-E) as well as with MO2 (Supplementary 167 Fig. $2 \mathrm{H}-\mathrm{I}$ ). Rescue experiments performed by co-injecting mouse Enpp4 mRNA (2 ng) with enpp4 MO1 168 or MO2 (10 ng each, $\mathrm{n}=72$ or 28 respectively) restore partially but significantly the normal phenotype 169 of s/c12a1 staining domain confirming the specificity of the knock-down of enpp 4 expression on 170 pronephric development (Fig. 2F and Fig. Supplementary 2J). Ectopic s/c12a1 expression was also 171 observed in some embryos (11\% and 50\% with MO1 or MO2 respectively) consistent with enpp4 over172 expression phenotype. The clcnkb domain and gata3 anterior expression domain were reduced after 173 enpp4 MO1 (53.5 \%, n = 40 and 31\%, n = 42 respectively) but these differences are not statistically 174 significant $\mathrm{p}>0.05$ ) (Fig. 2G-H). Enpp4 knock-down has no effect on glomus formation, as the expression 175 of wt1 and nphs1 was normal in most of enpp4 MO1 injected embryos at stage 33/34 (wt1= 94\%, n = 17634 and $n p h s 1=83 \%, n=41)($ Fig. 2l-J). These results suggest that enpp4 knock-down affected whole 177 pronephros differentiation rather than just the proximal-distal patterning of pronephric tubule 178 segmentation.

179 To address potential enpp4 roles during early phases of pronephros development, we tested by RT180 PCR its expression at key stages during kidney development in dissected developing pronephric tissues 181 (Fig. 3A). At later stages, enpp4 expression profile is in agreement with our published in situ 182 hybridization data ${ }^{16}$. However, weak expression is also detected in the embryonic kidney from stage 18312.5 and is upregulated by stage 26 . These data suggest that enpp4 might be involved during early 184 pronephric developmental phases. The expression domain of $I h x 1$ and pax 8 was altered following 185 knockdown of enpp4 by MO injection (See Supplementary Table 2 for raw data and statistical analyses). 186 At stage 28 , expression of $I h x 1$ was reduced especially in posterior elements of the pronephric anlagen $187(35 \%, \mathrm{n}=23$; Fig. $2 \mathrm{~K})$, although its expression in presumptive proximal tubules was sometimes 188 unaffected or expanded. At stage 24 , the expression domain of $I h \times 1$ was clearly reduced $(75 \%, n=20$, 189 Fig. 2L), suggesting involvement of enpp4 in early pronephros differentiation. Injection of enpp4 MO2 
caused similar phenotypes at both stages (stage $28,30 \%, n=20$; stage $24,55 \%, n=20$; Supplementary

Fig. 2K-L). At early neurula stages, expression of $I h x 1$ was also reduced following enpp4 MO1 or MO2 injection (MO1 37\%, n = 46; MO2 64\%, $n=61 ; p<0.001$; see also Supplementary Table 2) and even absent after MO1 injection (MO1 31\%, n = 46) (Fig. 2M and Supplementary Fig. 2M). The injection of both MO's resulted in a stronger reduction of $I h x 1$ expression $(n=31$; Fig. $2 \mathrm{~N}$ ). A reduction of pax8 expression was also observed at the neurula stages following enpp4 MO1 or MO2 injection (Fig. 20 and Supplementary Fig. 2N).

Taken together, the enpp4 knock-down and rescue experiments demonstrate that normal levels of enpp4 are required for normal pronephric development.

\section{Enpp4 misexpression phenotypes are distinct from those following enpp6 misexpression}

To address if the ectonucleotidase enpp6, also expressed in the proximal pronephric tubules ${ }^{16}$, can compensate for enpp4 loss of function, we performed single or double enpp4/enpp6 knock-down and rescue experiments (Supplementary Fig. 3 and Supplementary Table 2). Enpp6 depletion induced the formation of a smaller pronephros on the injected side, in the similar frequency than enpp4 knock-down (Supplementary Fig. 3B-C). The co-injection of enpp4 MO2 and enpp6 MO resulted in the formation of reduced $3 G 8(64 \%, n=76)$ and $4 A 6(62 \%, n=76)$ positive tissues and was not statistically different from the effects of enpp4 $\mathrm{MO} 2$, demonstrating that the enpp6 $\mathrm{MO}$ did not worsen the renal phenotype caused by enpp4 knock-down. Pronephric formation was altered following enpp6 over-expression, with reduced or absent, but never ectopic, pronephros observed and injection of enpp6 mRNA did not rescue the enpp4 MO2 phenotype (Supplementary Fig. 3D-E). Taken together, these data suggest that there is no functional redundancy between enpp4 and enpp6 ectonucleotidases.

Enpp4 misexpression affects expression of several components of the RA, Notch and Wnt signalling pathways.

Since Retinoic Acid (RA), Notch and Wnt signalling pathways are involved in pronephros formation and patterning $25,26,27,28,29,30$ and the timing of the endogenous expression of many components of these pathways overlap, we hypothesized that enpp4 might affect these pathways. We therefore examined embryos injected with enpp4 mRNA (Fig. 4A-G) or enpp4 MO1 (Fig. 4H-N) by in situ hybridization for alterations in expression domain of representative members e.g. ligands (dll1, jag1, wnt 4) receptors (notch1) and metabolic enzymes (raldh1a2, rdh10, cyp26a1) of these three pathways (see also Supplementary Table 3). Enpp4 over-expression induced ectopic and enlarged raldh1a2 and rdh10 expression domains in the pronephric region ( $p<0.001, n=37$ and 34 , respectively, Fig. 4A-B and Supplementary Table 3 ), while 
225 Supplementary Table 3). Raldh1a2 expression was unaffected in the pharyngeal arches. In contrast, 226 cyp26a1 expression was normal in the pronephric region following enpp4 mRNA or MO injection (98\%, $227 \mathrm{n}=44$ and 88\%, $\mathrm{n}=42$, respectively) (Fig. 4C, J). Both enpp4 mRNA and MO injection disturbed $r d h 10$ 228 and cyp26a1 expression in the somites. These results suggest enpp4 might act upstream of RA 229 signalling, potentially by controlling the expression of enzymes involved in RA synthesis. To verify this, 230 enpp4 expression, along with pax 8 and $l h \times 1$, was analysed in animal caps. Enpp4 expression is not 231 induced in animal caps treated with RA for three hours (Supplementary Fig. 4). This confirms the 232 epistatic relationship between enpp4 and RA signalling.

234 Enpp4 over-expression revealed significant enlarged expression domains of notch1 $(53 \% \mathrm{n}=75$, $235 \mathrm{p}<0.001$, Fig. 4D). Notch1 expression was normal in the majority of embryos after enpp4 knock-down $236(80 \%, \mathrm{n}=41$, Fig. 4K). Enpp4 mRNA injection caused ectopic (40\%) and enlarged (23\%) expression 237 domains of dll1 ( $\mathrm{n}=81, \mathrm{p}<0.001 \mathrm{Fig} .4 \mathrm{E}$ ), while MO injected embryos showed dll1 reduced expression 238 (33\%, $\mathrm{n}=46$, Fig. 4L). Ectopic (20\%) and enlarged (44\%) jag1 expression domains were observed 239 following enpp4 over-expression ( $n=81, p<0.001$, Fig. 4F), while MO1 injection reduced its expression 240 domain (in $38 \%$ of the analysed embryos, $n=42$, Fig. $4 M$ ). These results suggest that enpp4 also 241 regulates members of the Notch signalling pathway and that jag1 expression is more affected by enpp4 242 depletion than dll1 expression. Since rnfg over-expression caused ectopic pronephroi formation ${ }^{28}$, we 243 further addressed the link between enpp4 and the Notch pathway by injecting enpp4 mRNA or MO2 in 244 presence of $r$ fng mRNA or MO. Our data shows that modulation of notch-ligand interactions by fringe 245 proteins alters enpp4 pronephric phenotypes, although differences are not significant (Supplementary 246 Fig. 5 and Supplementary Table 3).

248 Finally, enpp4 mRNA injected embryos showed enlarged (32\%) and ectopic (17\%) wnt4 expression domains ( $n=41$, Fig. 4G) while expression of wnt4 was reduced in most of the enpp4 MO1 injected embryos (82\%, $n=39, p<0.001$ Fig. $4 \mathrm{~N}$, Supplementary Table 3). These data suggest that enpp4 is necessary and sufficient for promoting pronephric wnt4 expression.

\section{Xenopus enpp4 is localized to the plasma membrane}

255 To address the cellular localization of the amphibian enpp4 protein, we generated a specific polyclonal antibody against the full-length Xenopus protein (See Supplementary Table 4 for specificity evaluation of the antibody) and expressed Xenopus wild type (WT), T72S mutant and mouse Enpp4 cDNA in CHO cells by transient transfection. Xenopus enpp4 WT protein was detected, by western blotting, in whole 
260 experiments confirmed enpp4 expression at the cell membrane (Fig. 5B). These results show that 261 Xenopus enpp4 is a transmembrane protein, as its mouse ortholog ${ }^{31}$.

Phospholipid receptors are expressed in the developing pronephros along with the enpp4 gene members previously identified ${ }^{32}$ by RT-PCR in kidney dissected tissues (Fig. 3B-C). All Ipa receptors, except Ipar3 and 5, are expressed in pronephric tissues at a similar level from the time of kidney specification to late differentiation, confirming their ubiquitous expression profile during Xenopus embryogenesis ${ }^{32}$. The $s 1 p$ receptors display different expression profiles, with s1pr5 being the only family member to be expressed in the developing kidney at every stage analyzed, particularly in the presumptive pronephric tissue at stage 12.5. No such renal expression was detected by in situ hybridization in our previous study, although expression in marginal zone of blastula embryos was detected by RT-PCR ${ }^{32}$. These data suggest that the pronephric level of expression of these lipidic receptors, especially s1pr5, is relatively low, under the in situ hybridization detection level.

\section{Over-expression of s1pr5 enhances enpp4 function to induce ectopic pronephros}

In order to identify whether a lipidic receptor is involved in enpp4 phenotypes, s1pr and Ipar overexpression analyses were carried out by injecting 2ng of s1pr5, s1pr1, Ipa1.1 and p2y10 mRNAs alone or in combination with 1ng of enpp4 mRNA alone (Fig. 6A-D; Supplementary Fig. 6A-F; Supplementary Table 5) i.e half of the optimal dose to generate ectopic pronephros, Fig.1). At 1ng enpp4 mRNA dose ectopic 3G8 (7\%) and 4A6 (5\%) staining were obtained only in rare cases ( $n=94$, Fig. 6D) compared to the optimized dose of enpp4 mRNA used in Fig. 1. Injection of any tested lipidic receptor mRNA alone does not induce any ectopic kidney formation with normal 3G8 and 4A6 staining in the majority of the embryos ( $n=52$, Fig. 6C, Fig.S6B, D, F). Only co-expression of s1pr5 and enpp4 mRNAs resulted significantly in higher ectopic $3 G 8$ staining compared to enpp4 mRNA alone $(38 \%, n=89, p<0.001$, Fig. 


\section{The functions of enpp4 are mediated by the lipidic s1pr5 receptor}

296 To further confirm that s1pr5 is involved in pronephros development we performed loss of function 297 experiments. Two s1pr5 genes are identified in X.laevis genome and s1pr5.L corresponds to our 298 published sequence ${ }^{32}$. Despite distinct spatial expression in the adult frog, the two s1pr5 homeologs 299 display a very similar expression profile during X.laevis embryogenesis and are both expressed in the 300 pronephric tissues (Supplementary Fig. 7A,B). We therefore performed loss of function analyses with 301 an anti-sense MO against Xenopus s1pr5.L and s1pr5.S (See Supplementary Fig. 6G,H and 302 Supplementary Fig. 7C,D for MOs efficiency and specificity evaluation and Supplementary Table 5 for 303 raw data and statistical analyses). Embryos injected with 15ng of s1pr5.L MO or s1pr5.S MO displayed 304 significant reduced $3 G 8$ and $4 A 6$ staining $(n=43$ and 66 respectively, Fig. 6E-F, Supplementary Fig. 305 7E and Supplementary Table 5) suggesting that s1pr5 receptors are required for normal pronephros 306 formation in Xenopus.

307 To examine potential synergistic effects, we co-injected $7.5 \mathrm{ng}$ of s1pr5.L MO with $5 \mathrm{ng}$ of enpp4 MO 308 (half of the dose used previously for single injections) and compared their phenotype to those obtained 309 following co-injection of s1pr5 MO or enpp4 MO with control MO (Fig. 6G-J). As expected, embryos co310 injected with s1pr5.L and enpp4 MOs generated the strongest phenotype and smallest pronephros, with 311 strong reduction of $3 \mathrm{G} 8(74 \%)$ and 4A6 (81\%) staining domains $(\mathrm{n}=42$, Fig. $6 \mathrm{G}-\mathrm{H})$. Enpp4 MO alone 312 also caused strongly reduced 3G8 (65\%) and 4A6 (60\%) staining as previously shown $(n=40$, Fig. $6 \mathrm{~J})$, 313 while the s1pr5.L MO alone reduced pronephric size in both $3 G 8(22 \%)$ and 4A6 (29\%) domains less 314 frequently $(n=51$, Fig. 6I). Although there are no significant differences in pronephric phenotype 315 between enpp4 MO1+s1pr5.L MOs and enpp4 MO1 injected embryos, we concluded that co-injection 316 of s1pr5.L and enpp4 MOs showed additive effects on the inhibition of Xenopus pronephros 317 development based on the size of the scored pronephroi.

318 To further analyse the link between enpp4 and s1pr5, we carried out injection of 2ng of enpp4 mRNA 319 together with 15ng of s1pr5.L MO (Fig. 6K-L) or control MO. As expected, injection of s1pr5.L MO 320 lowered the percentage of embryos displaying ectopic $3 G 8$ and 4A6 staining caused by enpp4 mRNA 321 injection (17.5\% and $0 \%$ respectively, $n=40$, Fig. $6 \mathrm{~K}$; compared to $48 \%$ and $10 \%, n=50$, Fig. $6 \mathrm{~L}$ ).

322 Furthermore, the size and number per embryos of these ectopic pronephroi was lower than with injection 323 of $2 \mathrm{ng}$ of enpp4 and $15 \mathrm{ng}$ of cMO.

324 These results indicate that the ectopic pronephric tissues induced by enpp4 over-expression are due to 325 the activation of the s1pr5 receptor.

\section{Enpp4 specifically interacts with the lysophospholipid phosphatidylserine}

328 To assess if enpp4 hydrolyses lipids and generates a ligand, which could bind to the s1pr5 receptor, 329 phospholipid binding was tested by a protein lipid overlay assay using commercial pre-spotted lipid 
330 membranes. Out of the 26 bioactive lipids tested, only phosphatidylserine (PS) is specifically bound by 331 Xenopus enpp4 (Fig. 7A-B and Supplementary Fig. 8). Moreover, this interaction is abolished when the 332 putative catalytic site is mutated (Fig. 7C). We then tried to determine the enzymatic activity of Xenopus 333 enpp4. However, we could not detect any lipid derivatives, e.g DAG, PA or LysoPS, which could be 334 generated from the hydrolysis of PS in the membrane proteins fractions from over-expressing enpp4 $335 \mathrm{CHO}$ cells. Taken together, these data show enpp4 specifically interacts with PS but does not have 336 PLA, PLC or PLD activity towards PS.

\section{Discussion}

This paper reports newly identified and unexpected roles of the conserved ectonucleotidase enpp4 during vertebrate kidney development. Moreover, our findings provide a novel molecular mechanistic understanding for pronephric development and emphasizes the importance of the lipidic pathways in kidney formation (Fig. 8).

We previously showed that enpp4 is expressed in pronephric tubules ${ }^{16}$, but our present data demonstrate that low but significant levels of enpp4 can be detected at the time of proximal tubule specification ${ }^{33}$. Weak enpp4 expression was previously detected by RT-PCR but not by ISH, in gastrula embryo ${ }^{16}$. This discrepancy is attributable to the lower sensitivity of ISH compared to RT-PCR for the detection of gene expression patterns.

Our work demonstrates that enpp4 regulates the expression level of two of the transcription factors involved in pronephric anlagen formation, Ihx 1 and pax ${ }^{23}$ but not $i r x 1^{24}$. As $I h x 1$ is necessary for the early patterning of the entire kidney and subsequently growth and elongation in the development of the pronephric tubules ${ }^{34,35}$, the reduction of $I h x 1$ expression can explain the formation of the small pronephros in enpp4 morphants. Pax8 is necessary for the earliest steps of pronephric development and for pronephric precursors cell proliferation and can induce the formation of ectopic pronephric tubules ${ }^{23,36}$. Therefore, the ectopic expression of pax8 in enpp4 over-expressing embryos can explain the formation of ectopic pronephroi. We also demonstrate that enpp4 is sufficient to generate kidney, but only from lateral mesoderm and not in ectopic non-lateral positions. This suggests that the lateral mesoderm must contain either the receptor or the substrate necessary for enpp4 function. Furthermore, the induction of enpp4 expression in activin treated animal caps confirms the importance of mesoderm tissues for enpp4 pronephric functions (See Supplementary Fig.4). along their proximal/distal axis. Moreover, in some rare cases, there is a complete mini-duplication of the entire pronephros. This surprising phenotype could be explained by the up-regulation of the 
365 patterning of signalling pathway members. RA signaling is required during gastrulation for pronephric specification. Increased levels of RA signalling by enpp4 over-expression could lead to pax8 expression 367 activation and then to the formation of ectopic pronephroi ${ }^{23,25}$. RA signalling is also required post368 gastrulation for tubules morphogenesis and its down-regulation in enpp4 morphants could explain 369 tubules formation defects ${ }^{25}$. Subsequently, RA increase could pattern these ectopic tubules, as it has 370 been shown during zebrafish pronephric nephron segmentation patterning ${ }^{37,38}$. However, in Xenopus 371 pronephros, RA signalling increases expression level of distal tubules markers ${ }^{39}$. Moreover, RA 372 signalling also regulates the expression of members of the Notch pathway ${ }^{40}$, which can subsequently 373 activate wnt4 expression ${ }^{28}$, which then functions to pattern the proximal pronephros. Our data 374 demonstrate that rnfg protein is involved in mediating enpp4 signalling, probably by its ability to modify 375 Notch-ligand interactions ${ }^{41}$. Therefore, we speculate that enpp4 acts upstream or in parallel to RA 376 signalling and upstream of Notch and Wnt pathways (see Fig. 8). As enpp4 expression was unchanged 377 in animal caps treated with RA compared to control caps, this supports the hypothesis that enpp4 acts 378 upstream of the RA pathway.

379 A key question is how the mis-expression of enpp4, an ectonucleotidase, can alter gene expression. 380 Phosphatidylserine translocation across the cell membrane is a well-known indicator of apoptosis but is 381 also involved in physiological and developmental processes ${ }^{42,43}$. Therefore, enpp4 could bind to PS in 382 the extracellular space during pronephrogenesis. Enpp4 enzymatic activity is essential for ectopic 383 kidney formation, suggesting that renal alterations are due to an excess or shortage of enpp4 generated 384 products in the extracellular space inducing cell responses via the activation of the s1pr5 receptor. 385 However, our data strongly suggest that the enpp4 kidney phenotype is not linked to the bioactive lipids 386 LPA or S1P. The fact that the observed kidney phenotype might be due to a non-catalytic effect of enpp4 387 might be puzzling and unexpected, especially since enpp6 has been suggested to play major renal 388 physiological role through its enzymatic functions ${ }^{44}$. However, specific functions of other enpps, such 389 as enpp1, enpp2 and enpp5, have been shown to be independent of their enzymatic activity ${ }^{45,46,47,48 .}$ 390 It is therefore possible that enpp4 does not hydrolyze PS but its interaction with PS is necessary for the 391 activation of enpp4 and subsequently of s1pr5. PS binding and conformational change mechanisms 392 have been demonstrated for protein kinase C activation in mammalian kidney cells, supporting this 393 hypothesis ${ }^{49}$. Although we were unable to detect any of the predicted products of PS hydrolysis, we 394 cannot rule out that we failed to characterize enpp4 enzymatic activity and that enpp4 will generate a 395 bioactive lipid, other than S1P, which is able to bind to s1p5r, the most divergent member of the s1pr 396 family ${ }^{32}$. Although signaling through S1PR5 has been poorly studied, the activation of the S1P5 receptor 397 has been linked to an intracellular calcium increase and inactivation of the ERK pathway, both pathways 398 regulating pronephric field formation via RA signaling 50, 51, 52, 53, 54. 
399 We show that mouse Enpp4 can fulfil Xenopus enpp4 functions during pronephrogenesis, suggesting 400 mammalian kidney formation may be regulated by a similar mechanism demonstrated in this work. 401 ENPP4 is highly expressed in human metanephros and kidney tumours and its expression increases in 402 deceased donor kidney biopsies with delayed graft function after kidney transplantation (data from 403 human protein atlas ${ }^{55}$. Interestingly, ENPP4 is localized close to RUNX2 gene, whose mutations cause 404 cleidocranial dysplasia (CCD) ${ }^{56,57}$. Furthermore, a child with CDD and crossed renal ectopia has been 405 reported, and given our data, we can speculate that the ectopic kidney is attributable to ENPP4 locus 406 alterations ${ }^{58}$.

407 We propose a potentially novel model of action of the lipidic pathway in kidney physiology, implicating 408 either bioactive lipids distinct from LPA and S1P molecules or a novel non-catalytic interaction. The fact 409 that a S1P receptor might be activated other than by S1P binding may explain the polemic regarding 410 the beneficial actions of FTY720 in renal pathologies ${ }^{59}$. Moreover, our study raises potentially 411 fascinating possibilities regarding regenerative therapies for renal diseases. As therapies for acute renal 412 failure are still lacking, the identification of a novel pathway enabling the generation of ectopic kidneys 413 may provide useful insights to therapeutics that enhance human renal regeneration.

\section{Methods}

\section{Ethics Statement}

419 The work was carried out under a UK Home Office-approved animal procedures project licence and approved by the University of Warwick Biological Ethics Committee.

\section{Enpp4 Cloning and Site-Directed Mutagenesis}

423 The Xenopus enpp4 cDNA (Accession number: BC 079717) was cloned into pcDNA3.1. Mouse Enpp4 424 cDNA (Accession number: BC027749) was cloned into pCS2+ and pcDNA3.1. Site directed425 mutagenesis of the Xenopus enpp4 was performed by PCR using the Pfx polymerase (Invitrogen) and 426 adapted primers. The final PCR products were inserted into pCS2+ and pCDNA3.1 and verified by 427 sequencing.

\section{mRNA synthesis and morpholino oligonucleotides}

430 Capped mRNAs were synthesized using mMESSAGE mMACHINE Kits (Ambion) from linearized 431 plasmids. Plasmids used were Xenopus enpp4-pRNA3 (clone BC079717); mouse Enpp4-pCS2+; 432 Xenopus mutant enpp4-pCS2+; Xenopus s1p5r.L-pCS2+ (clone DC111014); Xenopus s1p1r-pCMV433 Sport6 (clone BC074356); Xenopus p2y10-pCMV-Sport6 (clone BC084356) and Xenopus rfng-pCMV- 
434 Sport $6^{28}$. enpp4 MO1 (5'-atgaaaacccttccaaacatcttga-3'), enpp4 MO2 (5'-gaaatgtcacacacgcagctcctat-

435 3'), enpp6 MO (5'-aacgtgctgtacttagccatgccac-3'), s1pr5.L MO (5'-catggttcgtcaatcctttatttc-3'), s1pr5.S $436 \mathrm{MO}\left(5^{\prime}\right.$-catggttcagtcaatgctttatctc-3'), rfng $\mathrm{MO}^{28}$ and standard control MO (cMO) were designed and 437 supplied by GeneTools, LLC.

438

439 Embryo culture, dissection, microinjections and lineage staining.

440 Xenopus embryos were staged according to ${ }^{60}$. Kidney and pronephric anlagen dissections were 441 performed in BarthX as previously described ${ }^{33}$. Each individual sample was injected into the lateral 442 marginal zone of a ventral-vegetal blastomere (V2) at the 8-cell stage to target the pronephros. Pilot 443 experiments were carried out to determine the enpp4 and lipidic receptors mRNAs and MOs quantities 444 to inject, based on their abilities to alter kidney development without affecting the overall morphology of 445 the embryos. The rfng mRNA and MO dose was used as previously published ${ }^{28}$. The LacZ (250pg) 446 mRNA was used as a lineage tracer. LacZ mRNA was injected alone or in combination with standard $447 \mathrm{MO}$ as controls. Injected embryos were cultured to various developmental stages, fixed in MEMFA and 448 stained for $\beta$-galactosidase activity (Red-Gal or X-Gal staining) to identify correctly targeted embryos. 449 Only embryos that had normal pronephros formation on the uninjected side and correctly targeted $\beta$ 450 galactosidase staining on the injected side were scored.

\section{Analysis of molecular marker expression in embryos}

453 Wholemount immunohistochemistry was performed using $3 G 8$ and 4A6 monoclonal antibodies as 454 previously described ${ }^{20}$. Whole-mount in situ hybridization was carried out as previously described ${ }^{61}$. 455 Anti-sense digoxigenin (DIG)-labelled RNA probes were synthesized from linearized template plasmids $45624,28,62$. Either BM purple (Roche Applied Science) or NBT/BCIP (Roche Applied Science) or Fast Red/ 457 Napthol AS/MX (Sigma) was used for the color reaction. After bleaching, embryos were photographed 458 with a magnification of $\times 10$ for whole stage 41 embryos, x20 for whole gastrula, neurula and early 459 organogenesis stages embryos and $x 32$ for pronephric region.

\section{Statistical analysis}

462 All experiments were repeated several times, on different batches of embryos, and pronephric 463 phenotypes were determined in a commonly used way, blind-coded, by comparing the injected and 464 uninjected sides ${ }^{28}$. The percentages of the embryos displaying the discussed phenotypes are given in 465 the text in bracket along with the total number of analysed embryos. All raw data and statistical analyses 466 are presented in the Tables (SI). Each histological analysis was numbered (see Supplementary Table $4671 \mathrm{~A}, 2 \mathrm{~A}, 3 \mathrm{~A}$ and $5 \mathrm{~A}$ ) and statistically pairwise compared as indicated in the Supplementary Table 1B, $4682 \mathrm{~B}, 3 \mathrm{~B}$ and $5 \mathrm{~B}$. Chi-square statistical analysis could not be performed for all comparison of experiments 
since the assumption was not always fulfilled. Hence, 2x5 Fisher's exact test was therefore used. Bonferroni multiple testing correction was then applied to all statistical analyses. All the statistical analyses were performed using the $\mathrm{R}$ statistical software Core Team $\mathrm{R}^{63}$.

\section{Acrylamide embedding, cryostat sectioning and Hoechst staining}

$474 X$. laevis embryos were embedded sectioned at $18 \mu \mathrm{m}$ thickness and nuclear Hoechst staining performed as described previously ${ }^{64}$.

\section{RT-PCR}

RT-PCR reactions were carried out on whole or dissected $X$. laevis embryos as described previously using the housekeeping gene odc as loading control. Quality of pronephric tissues dissections was assessed by amplification of the kidney markers $I h x 1$ and pax 8 and of the muscle marker myf5. Amplification conditions and primers sequences for the enpp, Ipar, s1pr, Ihx1 and pax8 genes have been previously published ${ }^{16,32,65}$. Myf5 was amplified using the forward primer, 5'-actactacagtctcccaggacaga3 ' and the reverse primer, 5'-agagtctggaatagggagggagca-3', with the annealing temperature of $60^{\circ} \mathrm{C}$ and 29 cycles. Each sample was analyzed in two independent embryo batches.

\section{Cell culturing and transient transfection}

487 Chinese hamster ovary (CHO) cells were cultured in HAMs F-12 (Gibco BRL) containing 10\% Foetal serum, $\mathrm{NaHCO}_{3}$ at $1.176 \mathrm{~g} / \mathrm{l}, 2 \mathrm{mM}$ of glutamine, $5 \mathrm{U} / \mathrm{ml}$ penicillin and $37.8 \mathrm{U} / \mathrm{ml}$ streptomycin during the 48 hours prior to transfection. Cells were then transfected for 24 hours using the reagent Turbofect (Fermentas) with $1 \mu \mathrm{g}$ of the eukaryotic constructs. The transfection medium was then removed and replaced with culture medium. Approximately $48 \mathrm{~h}$ post transfection, cells were fixed or harvested for analyses. As a control, $\mathrm{CHO}$ cells were transfected with empty vector pcDNA3.1.

\section{Anti-enpp4 antibody production}

495 The anti-XI enpp4 polyclonal antibody was raised in rabbits by direct intramuscular injection of the Xenopus laevis wild type enpp4-pcDNA3.1 plasmid followed by electroporation (Aldevron, LLC, USA). Rabbits were immunized 3 times, at day 0 , day 28 and 56 and terminal bleed performed at day 70 .

\section{Immunocytochemistry}

500 Immunocytochemistry was carried out on fixed unpermeabilized cells with polyclonal antibodies antiXlenpp4 used at 1/200 or anti-mEnpp4 (CR65; see ${ }^{31}$ ) used at 1/400 and anti-rabbit IgG FITC (Sigma) at $1 / 80$. The staining was recorded using a Nikon Optiphot/ Diginet camera system. Photographs were taken at a magnification of $X 40$. 


\section{Electrophoresis and Western Blot}

Native membrane proteins were extracted from transfected cells using the ProteoExtract ${ }^{\circ}$ Native Membrane Protein Extraction Kit (Calbiochem). Proteins from whole cells and from the membrane and soluble fractions were separated on a 12\% SDS-PAGE protein gel. Part of the gel was stained with Coomassie blue and processed for mass spectrometry analysis (Pôle Protéomique, Plateforme Génomique Fonctionnelle de Bordeaux, Université de Bordeaux) and the other was transferred onto a PVDF membrane (Bio-rad) overnight at $4^{\circ} \mathrm{C}$. To limit non-specific binding, the anti-Xlenpp4 antibody was pre-absorbed on untransfected $\mathrm{CHO}$ cells. The membrane was incubated overnight at $4^{\circ} \mathrm{C}$ with 1:200 dilution of enpp4 antiserum, washed and incubated in goat anti-rabbit IgG peroxidase secondary antibody (Sigma, dilution 1/2000) for 30 minutes at $20^{\circ} \mathrm{C}$. After several washes, immunoreactivity was detected by chemiluminescence (Western lighting Chemiluminescence Reagent Plus, Perkin Elmer).

\section{Lipid binding assay}

518 Hydrophobic membrane prespotted with bioactive lipids (ShingoStrips ${ }^{\mathrm{TM}} \mathrm{S}-6000$ and Membrane Lipids 519 Strips ${ }^{\mathrm{TM}}$ S-6002; Echelon Biosciences) were blocked 1 hour at $20^{\circ} \mathrm{C}$ with $1 \%$ BSA in Tris-buffered saline $520 \quad 0.05 \%$ Tween-20 (TBST). All subsequent washes were performed in TBST. Blots were overlaid with proteins extracts from membrane fractions of $\mathrm{CHO}$ cells transfected with enpp4-pcDNA3.1 or empty vector (dilution 1/30) in blocking buffer overnight at $4^{\circ} \mathrm{C}$. Membranes were washed and incubated with preabsorbed enpp4 serum (dilution 1/200) for 6 hours at room temperature. After several washes, the membranes were incubated with goat anti-rabbit IgG peroxidase secondary antibody for 30 minutes at room temperature, washed and developed using enhanced chemiluminescence. To confirm the observed binding, nitrocellulose Hybond-C extra (GE Healthcare) membranes were spotted with 0 to $200 \mu \mathrm{M}$ of PA (Sigma P-9511) or PS (Sigma P-6641) diluted into a mix of $\mathrm{MeOH} / \mathrm{CHCl}_{3} / \mathrm{H}_{2} \mathrm{O}(2 / 1 / 0.8$, $\mathrm{v} / \mathrm{v})$. Dried membranes were then treated as described above.

\section{References}

1. Desgrange A, Cereghini S. Nephron Patterning: Lessons from Xenopus, Zebrafish, and Mouse Studies. Cells 4, 483-499 (2015).

2. Jones EA. Xenopus: a prince among models for pronephric kidney development. J Am Soc Nephrol 16, 313-321 (2005).

3. Lienkamp SS. Using Xenopus to study genetic kidney diseases. Semin Cell Dev Biol 51, 117-124 (2016).

4. Wessely O, Tran U. Xenopus pronephros development--past, present, and future. Pediatr Nephrol 26, 1545-1551 (2011). 
5. Krneta-Stankic V, DeLay BD, Miller RK. Xenopus: leaping forward in kidney organogenesis. Pediatr Nephrol 32, 547-555 (2017).

6. Solini A, Usuelli V, Fiorina P. The dark side of extracellular ATP in kidney diseases. J Am Soc Nephrol 26, 1007-1016 (2015).

7. Park F, Miller DD. Role of lysophosphatidic acid and its receptors in the kidney. Physiol Genomics 49, 659-666 (2017).

8. Huwiler A, Pfeilschifter J. Sphingolipid signaling in renal fibrosis. Matrix Biol 68-69, 230-247 (2018).

9. Burnstock G, Evans LC, Bailey MA. Purinergic signalling in the kidney in health and disease. Purinergic Signal 10, 71-101 (2014).

10. Kirby RJ, Jin Y, Fu J, Cubillos J, Swertfeger D, Arend LJ. Dynamic regulation of sphingosine-1-phosphate homeostasis during development of mouse metanephric kidney. Am J Physiol Renal Physiol 296, F634641 (2009).

11. Kihara Y, Maceyka M, Spiegel S, Chun J. Lysophospholipid receptor nomenclature review: IUPHAR Review 8. Br J Pharmacol 171, 3575-3594 (2014).

12. Burnstock G. Purine and purinergic receptors. Brain Neurosci Adv 2, 2398212818817494 (2018).

13. Zimmermann $\mathrm{H}$, Zebisch $\mathrm{M}$, Strater N. Cellular function and molecular structure of ecto-nucleotidases. Purinergic Signal 8, 437-502 (2012).

14. Dwyer KM, Kishore BK, Robson SC. Conversion of extracellular ATP into adenosine: a master switch in renal health and disease. Nat Rev Nephrol 16, 509-524 (2020).

15. Stefan C, Jansen S, Bollen M. NPP-type ectophosphodiesterases: unity in diversity. Trends Biochem Sci 30, 542-550 (2005).

16. Massé K, Bhamra S, Allsop G, Dale N, Jones EA. Ectophosphodiesterase/nucleotide phosphohydrolase (Enpp) nucleotidases: cloning, conservation and developmental restriction. Int J Dev Biol 54, 181-193 (2010).

17. Albright RA, et al. NPP4 is a procoagulant enzyme on the surface of vascular endothelium. Blood 120, 4432-4440 (2012).

18. Albright RA, et al. Molecular basis of purinergic signal metabolism by ectonucleotide pyrophosphatase/phosphodiesterases 4 and 1 and implications in stroke. J Biol Chem 289, 3294-3306 (2014).

19. Yan D, et al. Homology modeling and docking studies of ENPP4: a BCG activated tumoricidal macrophage protein. Lipids Health Dis 15, 19 (2016).

20. Vize PD, Jones EA, Pfister R. Development of the Xenopus pronephric system. Dev Biol 171, 531-540 (1995).

21. Raciti D, et al. Organization of the pronephric kidney revealed by large-scale gene expression mapping. Genome Biol 9, R84 (2008).

22. Reggiani L, Raciti D, Airik R, Kispert A, Brandli AW. The prepattern transcription factor Irx3 directs nephron segment identity. Genes Dev 21, 2358-2370 (2007).

23. Carroll TJ, Vize PD. Synergism between Pax-8 and lim-1 in embryonic kidney development. Dev Bio/ 214, 46-59 (1999). 
630

631

632

633

634

635

636

637

638

639

640

641

642

643

644

645

646

647

648

649

650

651

652

653

654

655

656

657

658

659

24. Alarcon P, Rodriguez-Seguel E, Fernandez-Gonzalez A, Rubio R, Gomez-Skarmeta JL. A dual requirement for Iroquois genes during Xenopus kidney development. Development 135, 3197-3207 (2008).

25. Cartry $\mathrm{J}$, et al. Retinoic acid signalling is required for specification of pronephric cell fate. Dev Biol 299, 35-51 (2006).

26. Lyons JP, et al. Requirement of Wnt/beta-catenin signaling in pronephric kidney development. Mech Dev 126, 142-159 (2009).

27. McLaughlin KA, Rones MS, Mercola M. Notch regulates cell fate in the developing pronephros. Dev Biol 227, 567-580 (2000).

28. Naylor RW, Jones EA. Notch activates Wnt-4 signalling to control medio-lateral patterning of the pronephros. Development 136, 3585-3595 (2009).

29. Saulnier DM, Ghanbari H, Brandli AW. Essential function of Wnt-4 for tubulogenesis in the Xenopus pronephric kidney. Dev Biol 248, 13-28 (2002).

30. Taelman V, Van Campenhout C, Solter M, Pieler T, Bellefroid EJ. The Notch-effector HRT1 gene plays a role in glomerular development and patterning of the Xenopus pronephros anlagen. Development 133, 2961-2971 (2006).

31. Moller S, et al. Monitoring the expression of purinoceptors and nucleotide-metabolizing ecto-enzymes with antibodies directed against proteins in native conformation. Purinergic Signal 3, 359-366 (2007).

32. Massé K, Kyuno J, Bhamra S, Jones EA. The lysophosphatidic acid (LPA) and sphingosine-1-phosphate (S1P) receptor gene families: cloning and comparative expression analysis in Xenopus laevis. Int J Dev Biol 54, 1361-1374 (2010).

33. Brennan HC, Nijjar S, Jones EA. The specification of the pronephric tubules and duct in Xenopus laevis. Mech Dev 75, 127-137 (1998).

34. Chan TC, Takahashi S, Asashima M. A role for Xlim-1 in pronephros development in Xenopus laevis. Dev Biol 228, 256-269 (2000).

35. Cirio MC, et al. Lhx1 is required for specification of the renal progenitor cell field. PLoS One 6, e18858 (2011).

36. Buisson I, Le Bouffant R, Futel M, Riou JF, Umbhauer M. Pax8 and Pax2 are specifically required at different steps of Xenopus pronephros development. Dev Biol 397, 175-190 (2015).

37. Naylor RW, Skvarca LB, Thisse C, Thisse B, Hukriede NA, Davidson AJ. BMP and retinoic acid regulate anterior-posterior patterning of the non-axial mesoderm across the dorsal-ventral axis. Nat Commun 7 , 12197 (2016).

38. Wingert RA, et al. The cdx genes and retinoic acid control the positioning and segmentation of the zebrafish pronephros. PLoS Genet 3, 1922-1938 (2007).

39. Van Campenhout C, et al. Evi1 is specifically expressed in the distal tubule and duct of the Xenopus pronephros and plays a role in its formation. Dev Biol 294, 203-219 (2006).

40. Arima K, et al. Global analysis of RAR-responsive genes in the Xenopus neurula using cDNA microarrays. Dev Dyn 232, 414-431 (2005).

41. Haines N, Irvine KD. Glycosylation regulates Notch signalling. Nat Rev Mol Cell Biol 4, 786-797 (2003).

42. Leventis PA, Grinstein S. The distribution and function of phosphatidylserine in cellular membranes. Annu Rev Biophys 39, 407-427 (2010). 
43. van den Eijnde SM, et al. Transient expression of phosphatidylserine at cell-cell contact areas is required for myotube formation. J Cell Sci 114, 3631-3642 (2001).

44. Sakagami $\mathrm{H}$, et al. Biochemical and molecular characterization of a novel choline-specific glycerophosphodiester phosphodiesterase belonging to the nucleotide pyrophosphatase/phosphodiesterase family. J Biol Chem 280, 23084-23093 (2005).

45. Belfiore A, et al. Overexpression of membrane glycoprotein PC-1 in MDA-MB231 breast cancer cells is associated with inhibition of insulin receptor tyrosine kinase activity. Mol Endocrinol 10, 1318-1326 (1996).

46. Dennis J, Nogaroli L, Fuss B. Phosphodiesterase-lalpha/autotaxin (PD-lalpha/ATX): a multifunctional protein involved in central nervous system development and disease. J Neurosci Res 82, 737-742 (2005).

47. Grupe A, Alleman J, Goldfine ID, Sadick M, Stewart TA. Inhibition of insulin receptor phosphorylation by PC-1 is not mediated by the hydrolysis of adenosine triphosphate or the generation of adenosine. $J$ Biol Chem 270, 22085-22088 (1995).

48. Ohe $\mathrm{Y}$, et al. Characterization of nucleotide pyrophosphatase-5 as an oligomannosidic glycoprotein in rat brain. Biochem Biophys Res Commun 308, 719-725 (2003).

49. Bell RM, Hannun YA, Loomis CR. Mechanism of regulation of protein kinase $C$ by lipid second messengers. Symp Fundam Cancer Res 39, 145-156 (1986).

50. Futel M, et al. TRPP2-dependent Ca2+ signaling in dorso-lateral mesoderm is required for kidney field establishment in Xenopus. J Cell Sci 128, 888-899 (2015).

51. Im DS, et al. Characterization of a novel sphingosine 1-phosphate receptor, Edg-8. J Biol Chem 275, 14281-14286 (2000).

52. Le Bouffant R, Wang JH, Futel M, Buisson I, Umbhauer M, Riou JF. Retinoic acid-dependent control of MAP kinase phosphatase-3 is necessary for early kidney development in Xenopus. Biol Cell 104, 516-532 (2012).

53. Leclerc $\mathrm{C}$, Webb SE, Miller AL, Moreau M. An increase in intracellular $\mathrm{Ca} 2+$ is involved in pronephric tubule differentiation in the amphibian Xenopus laevis. Dev Biol 321, 357-367 (2008).

54. Sanchez T, Hla T. Structural and functional characteristics of S1P receptors. J Cell Biochem 92, 913-922 (2004).

55. Mas VR, et al. Gene expression patterns in deceased donor kidneys developing delayed graft function after kidney transplantation. Transplantation 85, 626-635 (2008).

56. Cohen MM, Jr. Perspectives on RUNX genes: an update. Am J Med Genet A 149A, 2629-2646 (2009).

57. Glusman G, Kaur A, Hood L, Rowen L. An enigmatic fourth runt domain gene in the fugu genome: ancestral gene loss versus accelerated evolution. BMC Evol Biol 4, 43 (2004).

58. Suresh SS. A family with cleidocranial dysplasia and crossed ectopic kidney in one child. Acta Orthop Belg 75, 521-527 (2009).

59. Jo SK, Bajwa A, Awad AS, Lynch KR, Okusa MD. Sphingosine-1-phosphate receptors: biology and therapeutic potential in kidney disease. Kidney Int 73, 1220-1230 (2008).

60. Faber J, Nieuwkoop PD. Normal Table of Xenopus Laevis. Garland Publishing Inc (1994).

61. Harland RM. In situ hybridization: an improved whole-mount method for Xenopus embryos. Methods Cell Biol 36, 685-695 (1991). 


\section{Abbreviations}

734 Enpp: ectophosphodiesterase/nucleotide phosphohydrolases; LPA: lysophosphatidic acid; MO: morpholino-oligonucleotide; PA: phosphatidic acid; PLA, phospholipase A; PLC, phospholipase C; PLD, phospholipase D; PS: phosphatidylserine; RA: retinoic acid; S1P: sphingosine-1-phosphate.

\section{Acknowledgments}

We are grateful to Junichi Kyuno for his contribution to experimental design and data analysis. We thank for maintenance of frogs. We also thank J-W. Dupuy (Pôle Protéomique Plateforme Génomique

742 Fonctionnelle Bordeaux, Université de Bordeaux) for MS analysis, and B. Arveiler for helpful comments 743 regarding human pathologies. The work was supported by Wellcome trust grant 082071 as well as 744 CNRS and University of Bordeaux.

\section{Author Contributions}

$747 \mathrm{KM}$, and EAJ designed research; KM, EAJ, SB, LM-P, performed research; KM, EAJ, CP, EB-G, LM-P analysed data; KM and EAJ wrote the paper with contributions from all the authors.

\section{Competing Interest}

751 The authors declare no competing interests.

752

\section{Materials \& Correspondence}

754 Materials requests should be addressed to the corresponding author KM, karine.masse@u-bordeaux.fr 

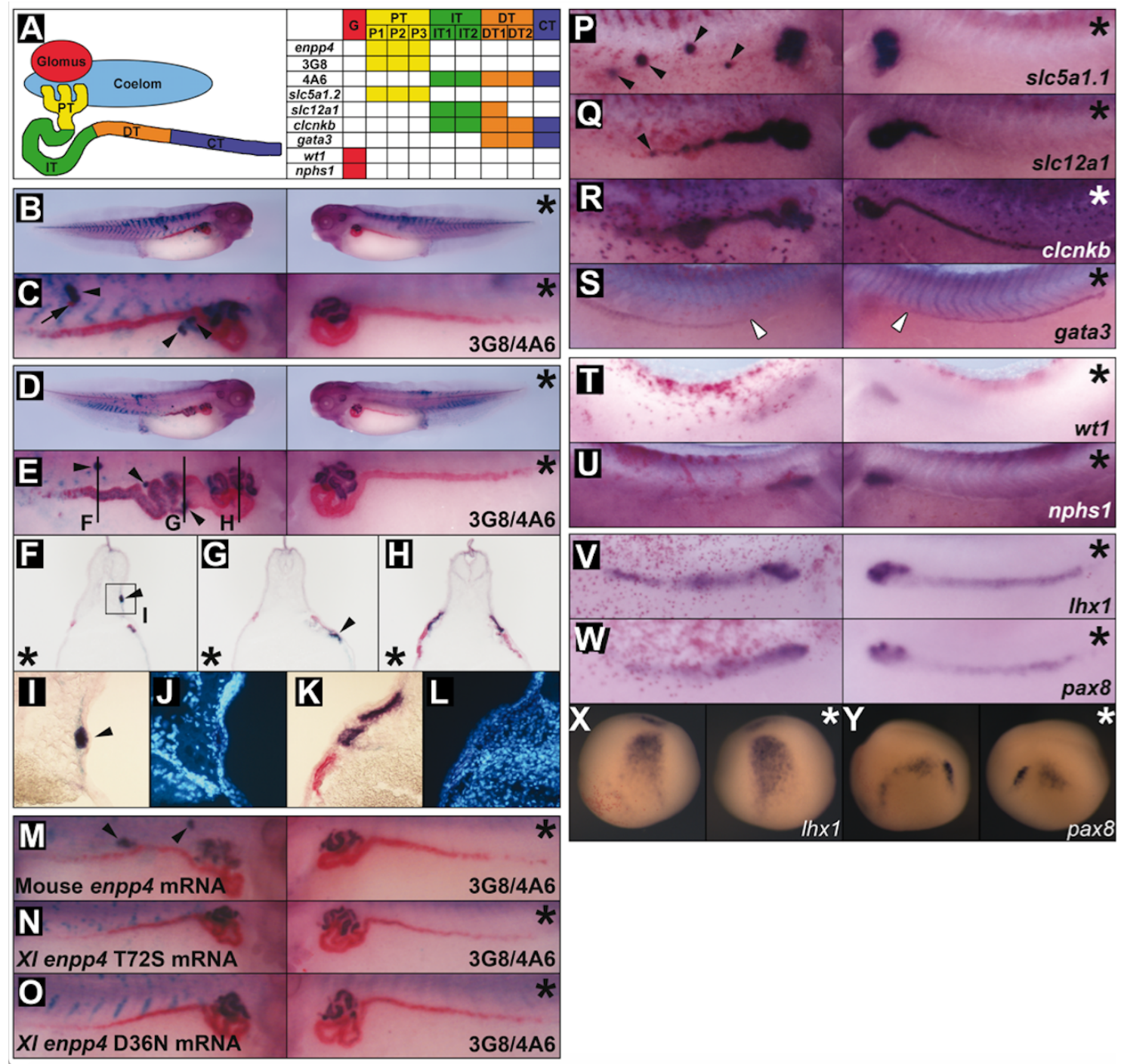

Fig. 1. Over-expression of enpp4 induces ectopic proximal pronephric tubules. (A) Schematic diagram of pronephric structural components showing the expression domain for each marker used in this study, adapted from ${ }^{20}$. G: glomus, PT: proximal tubule, IT: intermediate tubule, DT: distal tubule, CT: collecting tubule. (B-Y) Embryos injected with 2ng of enpp4 and 250pg of LacZ mRNAs were examined by 3G8/4A6 antibody staining (B-O) or whole mount in situ hybridization with the following probes: slc5a1.1 (P), s/c12a1 (Q), clcnkb (R) and gata3 (S) at stage 37/38; wt1 (T) and nphs1 (U) at stage 32; Ihx1 (V, X) and pax8 (W, Y) at stages 28 and 14. (F-L) Transverse sections of the embryo shown in panels $D$ and $E$ were cut in the anterior-posterior registers indicated by lines in panel $E$. A higher magnification image (I) of ectopic pronephros in the somite indicated by square in $(F)$ and of control kidney $(\mathrm{K})$ and counterstained with Hoechst to indicate nuclei $(\mathrm{J}, \mathrm{L})$. Embryos injected with $2 \mathrm{ng}$ of mouse wild type Enpp4 (M), X.laevis mutated in the putative catalytic site $(\mathrm{N})$ or in the cation binding site $(O)$ and $250 \mathrm{pg}$ of $L a c Z$ mRNAs were examined by $3 G 8 / 4 A 6$ antibody staining. The asterisk denotes the uninjected side of each embryo. Arrowheads indicate ectopic marker staining. Blank arrowheads in $S$ indicate the anterior limit of gata3 expression. See also Supplementary Table 1 for raw data and statistical analyses and Supplementary Fig. 1. 


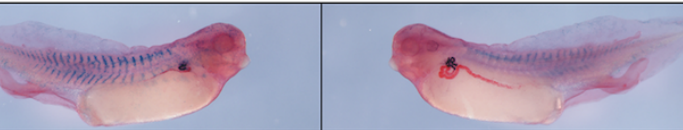

B

\section{M01+2}

\section{D}

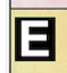

目

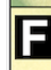

\section{+2ng mRNA}

G

H

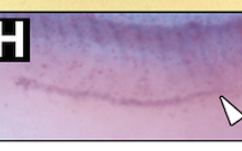

I

3G8/4A6

J

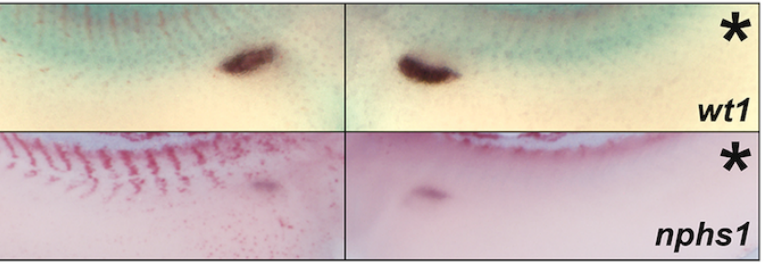

K

$3 \mathrm{G} 8 / 4 \mathrm{~A} 6$

* $\mathrm{L}$

slc5a1.1

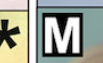

$\mathbf{M}-* \mathbf{N}$

Ihx1 St.24

Ihx1 St.28

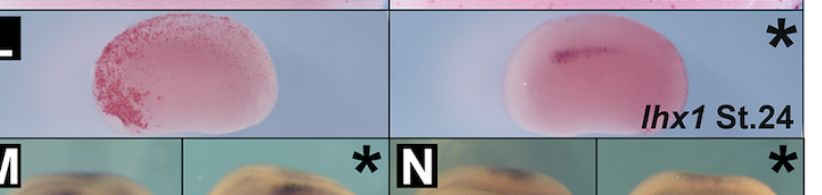

783

784

785

786

787

788

789

790

791

792

793

Fig. 2. MO knockdown of enpp4 expression disrupts pronephros formation. Embryos targeted with $10 \mathrm{ng}$ of enpp4 MO1 or $10 \mathrm{ng}$ of both enpp4 MOs (C, N) and 250pg of lacZ mRNA were examined by 3G8/4A6 antibody staining (A-C) or whole mount in situ hybridization with the following probes: s/c5a1.1 (D), slc12a1 (E-F), clcnkb (G) and gata3 (H) at stage 37/38; wt1 (I) and nphs1 (J) at stage 32, Ihx1 (K$\mathbf{N})$ at stages 28, 24 and 14, pax8 (O) at stage 14. The embryo shown in (F) was co-injected with 2 ng of mouse Enpp4 mRNA to rescue enpp4 knock-down phenotype. The asterisk denotes the control, uninjected side of each embryo. Blank arrowheads in $(\mathrm{H})$ indicate the anterior limit of gata3 expression. see also Supplementary Table 2 for raw data and statistical analyses and Supplementary Fig. 2 and S3. 
A

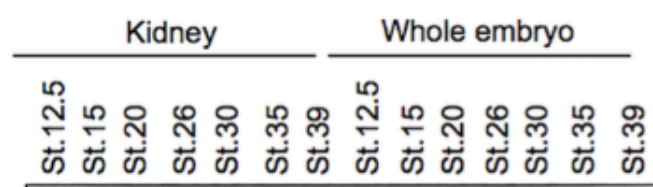

\begin{tabular}{|c|c|c|c|c|}
\hline enpp1 & - & - & --- & - \\
\hline enpp2a & -- & $=$ & --- & - \\
\hline enpp2b & ---1 & $=$ & ---- & -- \\
\hline enpp3 & -- & $\overline{-}$ & ----1 & - \\
\hline enpp4 & --- & - & -- & -- \\
\hline enpp6 & - & - & & 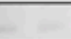 \\
\hline
\end{tabular}
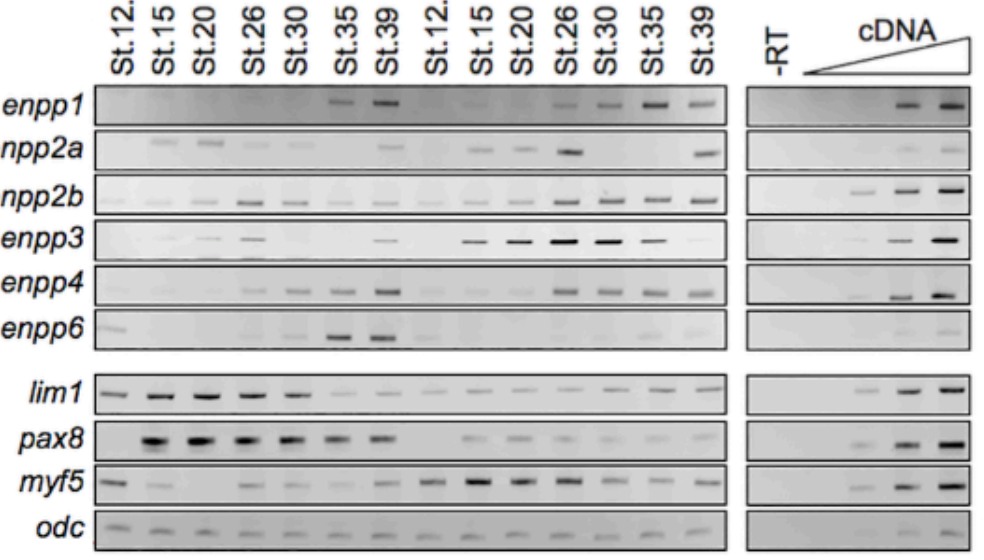

B
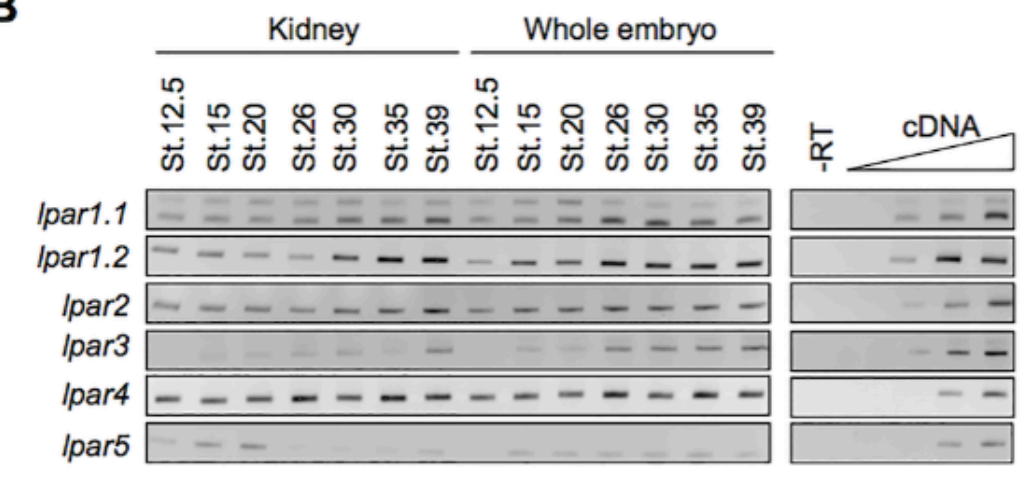

C
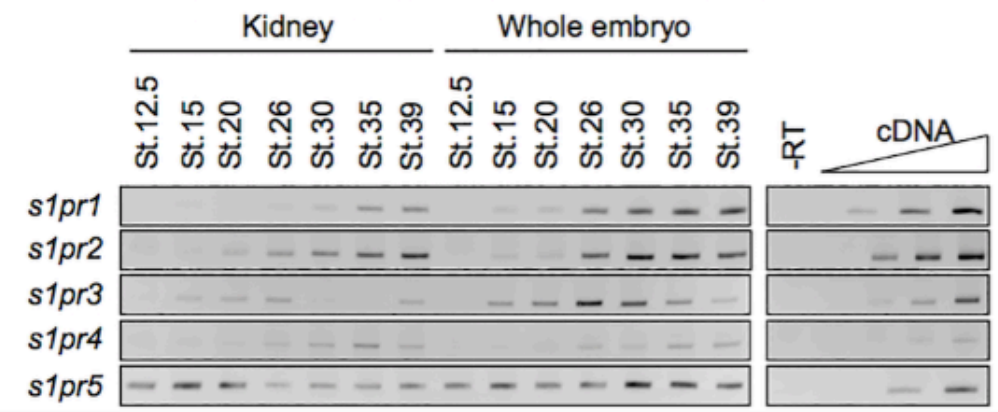

Fig. 3. The enpp and the lipidic receptors, the Ipar and s1pr, gene family members are expressed in the pronephros. Developing pronephric anlagen or pronephric tubules were dissected as indicated, from whole $X$. laevis embryos and total RNA extracted. RT-PCR was performed on pronephric dissected tissues and control whole embryos along with negative and linearity controls. (A) Comparative expression pattern of the enpp genes and pronephric and muscle marker genes controlling the quality of the dissections (B) Comparative expression profile of the Ipa receptors (C) Comparative expression profile of the s1prgenes. 
809

810

811

812

813

814

815

816

817

818

819

820

821

822

823

824

825
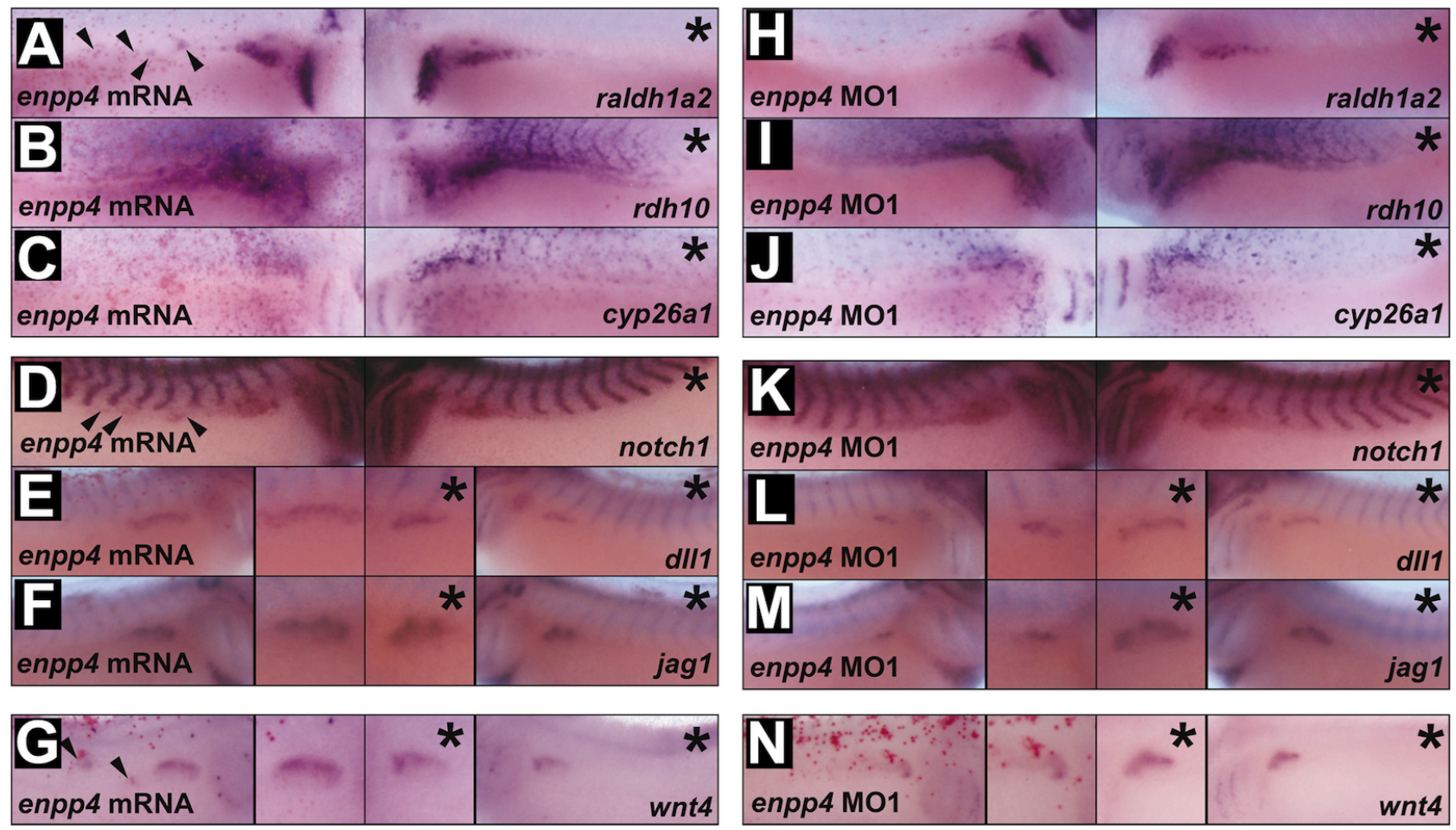

Fig. 4. Microinjection of enpp4 mRNA and MO affect the expression of retinoic acid synthesis enzymes, notch and wnt signalling molecules. (A-G) Embryos targeted with 2ng of enpp4 and 250pg of LacZ mRNA or (H-N) 10ng of enpp4 MO and 250pg of LacZ mRNA were fixed at stage 28 and examined by whole mount in situ hybridization with the following probes: raldh1a2 (A, H), rdh10 (B, I), cyp26a1 (C, J), notch1 (D, K), dll1 (E, L), jag1 (F, M) and wnt4 (G, N). The asterisks denote the control, uninjected sides. Arrowheads indicate ectopic staining of the marker gene (see also Supplementary Table 3 and Supplementary Fig. 4). 

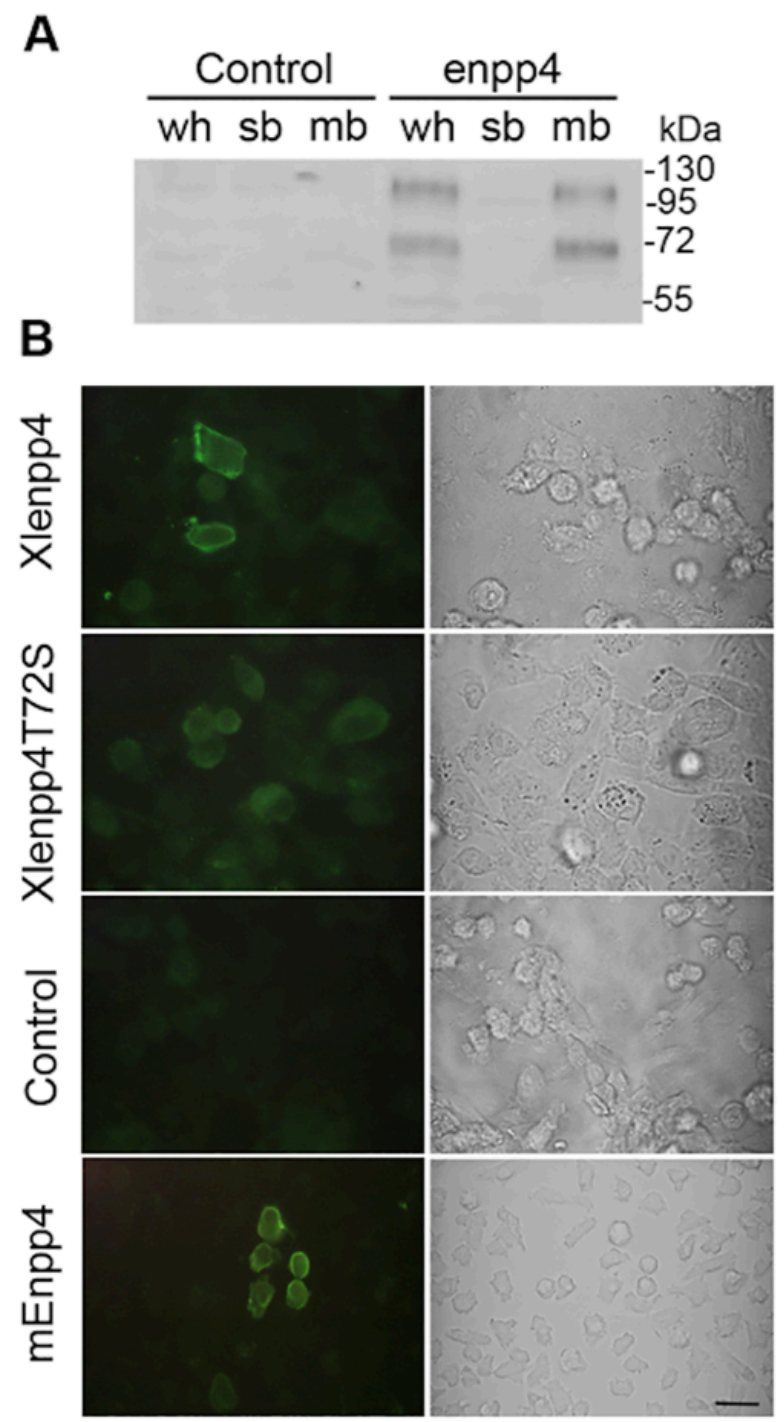

831 Fig. 5. Enpp4 is a transmembrane ectonucleotidase. (A) CHO cells were transfected with Xlenpp4832 pcDNA3.1 or empty vector (control) and the cellular distribution of enpp4 determined by western blotting 833 using anti-Xlenpp4 antibody and proteins extracts from membrane ( $\mathrm{mb}$ ), soluble (sb) or whole cells (wh) 834 fractions. (B) Representative images of the cellular distribution of enpp4 determined by 835 immunofluorescence using anti-Xlenpp4 or anti-mEnpp4 antibodies from $\mathrm{CHO}$ cells transfected with 836 Xlenpp4-pcDNA3.1, Xlenpp4T72S-pcDNA3.1, mEnpp4-pcDNA3.1 or empty pcDNA3.1 vector (control). 837 Corresponding brightfield images are also presented. Scale bar represents $20 \mu \mathrm{m}$. 

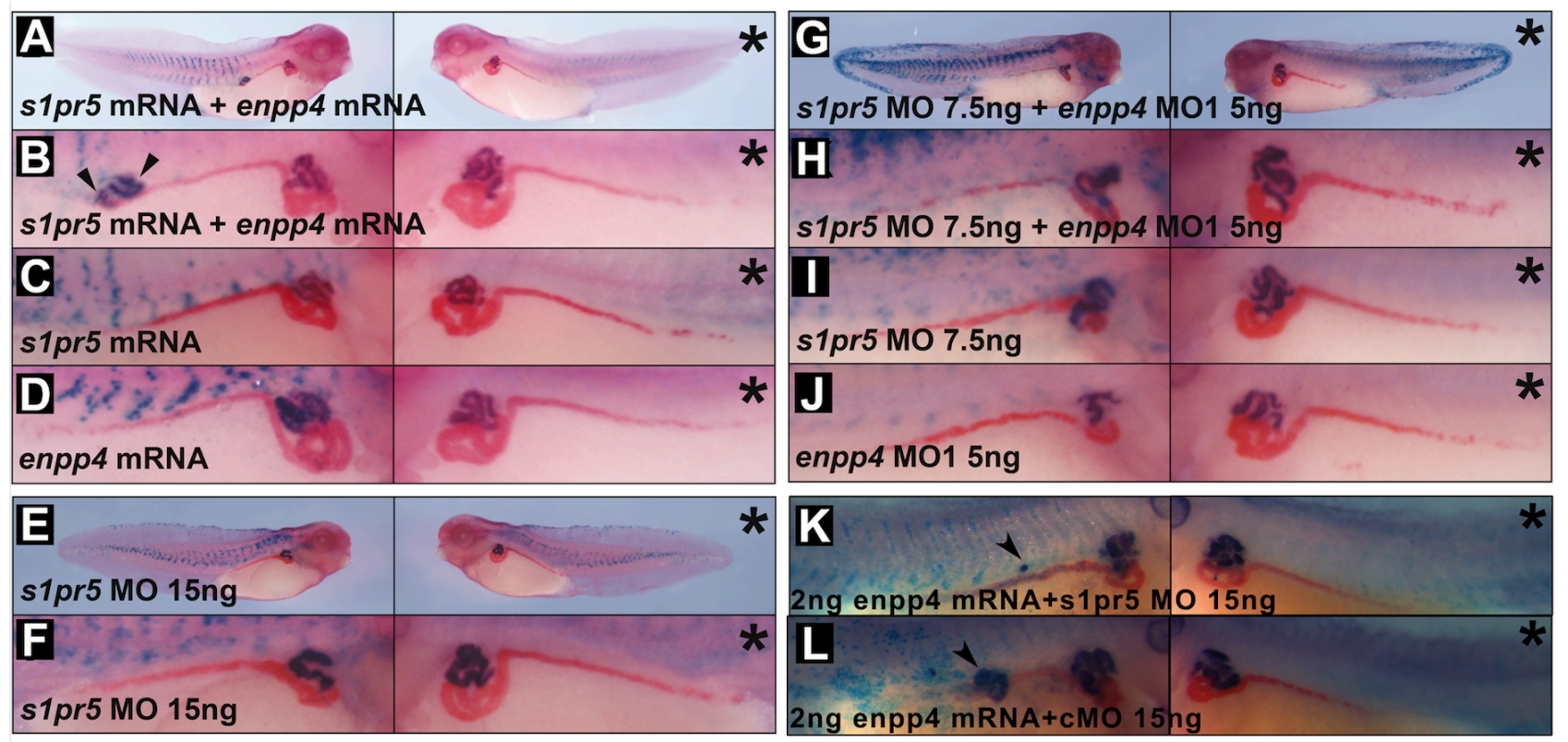

Fig. 6. Enpp4 pronephric functions are mediated by the s1pr5 receptor. Injected embryos were examined by 3G8/4A6 antibody staining following (A, B) double targeted injection of 2ng s1pr5.L mRNA and $1 \mathrm{ng}$ of enpp4 mRNA, (C) single targeted injection of s1pr5.L (D) enpp4 mRNA or (E-F) embryos injected with $15 \mathrm{ng}$ of s1pr5.L MO. (G-H) Double targeted injection of $7.5 \mathrm{ng}$ of s1pr5.L MO and $5 \mathrm{ng}$ of enpp4 MO1. (I, J) Single targeted injection of s1pr5.L MO (I) or enpp4 MO1 (J). (K, L) Double targeted injection of $15 \mathrm{ng}$ of s1pr5.L MO and 2ng of enpp4 mRNA (K) and 15ng of cMO and 2ng of enpp4 mRNA (L). An asterisk denotes the control uninjected side. An arrowhead indicates ectopic $3 \mathrm{G} 8$ staining. see also Supplementary Table 5 and Supplementary Fig. 7 and S6. 
A

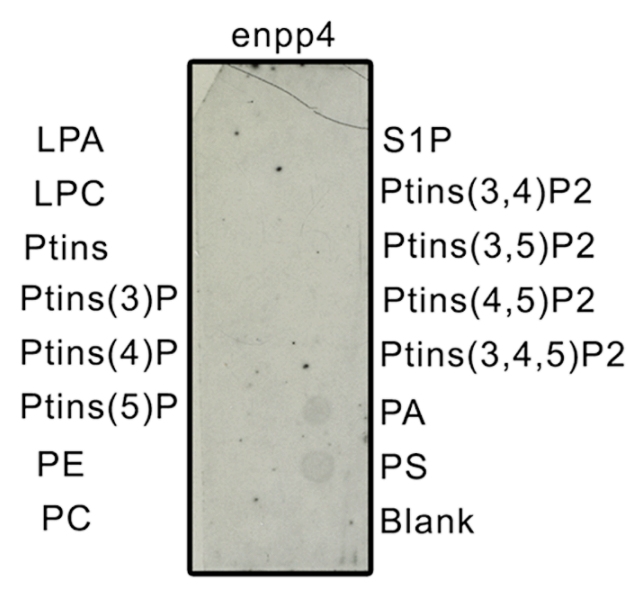

B

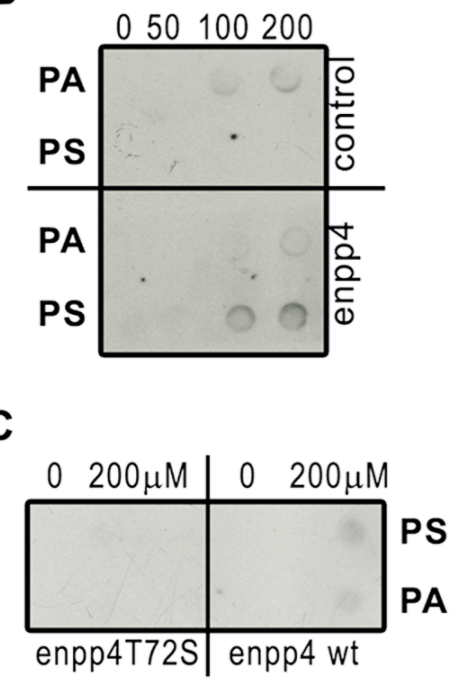

Fig. 7. Enpp4 specifically binds to the lysophospholipid, phosphatidylserine. (A) Membrane Lipid strip $^{\mathrm{TM}}$ was incubated with membrane protein extracts from enpp4 over-expressing $\mathrm{CHO}$ cells and the bound enpp4 protein detected with anti-Xlenpp4 serum. (B, C) Nitrocellulose membranes were spotted with increasing amount of PA or PS and incubated with membrane protein extracts from $\mathrm{CHO}$ cells transfected with enpp4-pcDNA3.1, with enpp4T72S-pcDNA3.1 or empty plasmid (control) and the bound proteins detected with anti-Xlenpp4 serum. LPA, lysophosphatidic acid; LPC, lysophosphocholine; PA, phosphatidic acid; PC, phospatidylcholine; PE, phosphatidylethanolamine; PS, phosphatidylserine; PtIns, phosphatidylinositol; S1P, shingosine-1-phosphate. See also Supplementary Fig. 7. 
A

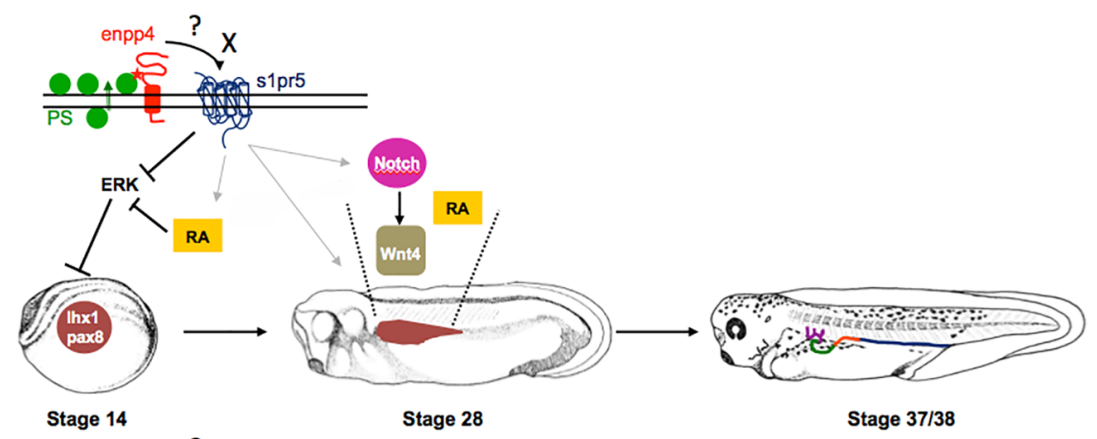

B

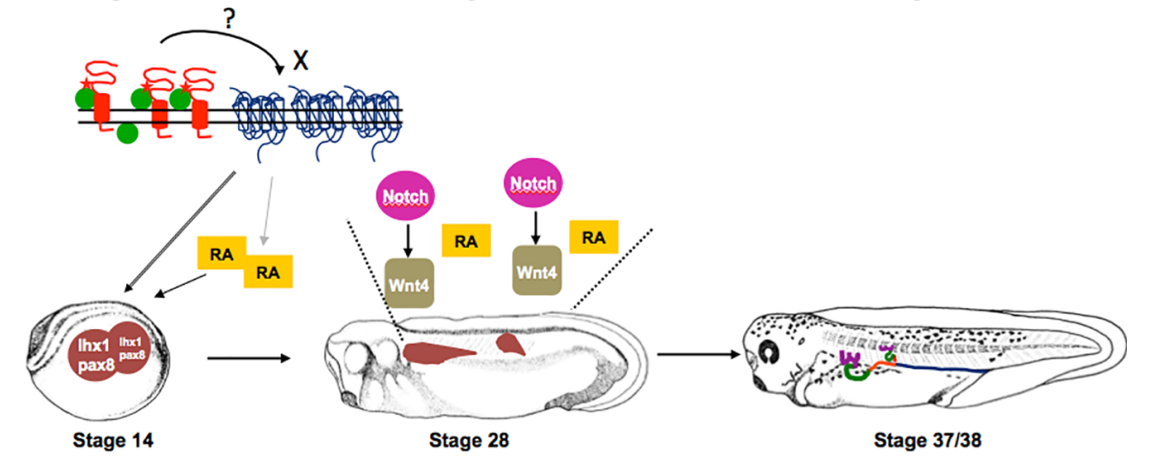
pronephric development, in the extracellular space, enpp4 binds to phosphatidylserine close to, or in its catalytic site, which can then either interact with the s1pr5 or produce a novel ligand $X$, able to bind to this receptor. The activation of s 1 pr5 leads to the up-regulation of $I h \times 1 /$ pax 8 pronephric markers in the kidney field either by acting upstream of RA signalling pathway or by acting directly via the ERK or calcium pathways. At later stages, RA is required for tubules morphogenesis and Notch and Wnt pathway are involved in the patterning of the pronephric tubules. The mechanism by which s1pr5 activation directs the expression domains of these genes remains to be confirmed. (B) Enpp4 and s1pr5 over-expression leads to expanded and ectopic expression domains for both the Notch and RA pathway genes and wnt4. These changes in patterning gene expression domains induce the formation of enlarged pronephric segments and ectopic pronephric tubules. 